\title{
A novel plant actin-microtubule bridging complex regulates cytoskeletal and ER structure at Endoplasmic Reticulum-Plasma Membrane Contact Sites (EPCS)
}

Jingze Zang ${ }^{1,2,6}$, Sandra Klemm ${ }^{3,6}$, Charlotte Pain ${ }^{4}$, Patrick Duckney ${ }^{2}$, Zhiru Bao ${ }^{1}$, Gina Stamm ${ }^{3}$, Verena Kriechbaumer ${ }^{4}$, Katharina Bürstenbinder ${ }^{3}$, Patrick J Hussey ${ }^{2,5 *}$ and Pengwei Wang ${ }^{1 *}$

1. Key Laboratory of Horticultural Plant Biology (MOE), College of Horticulture and Forestry Sciences, Huazhong Agricultural University, Wuhan 430070, Hubei Province, China; 2. Department of Biosciences, Durham University, South road, Durham, DH1 3LE, UK; 3. Department of Molecular Signal Processing, Leibniz Institute of Plant Biochemistry, Halle (Saale), Germany; 4. Plant Cell Biology, Oxford Brookes University, Oxford, OX3 OBP, UK; 5, Department of Experimental Plant Biology, Charles University, Faculty of Science, Viničná 5, CZ 12843 Praha 2, Czechia

6. These authors contributed equally to this work

*Corresponding author: wangpengwei@mail.hzau.edu.cn (Lead Contact); p.j.hussey@durham.ac.uk

\section{Summary}

In plants, the cortical ER network is connected to the plasma membrane through the ER-PM contact sites (EPCS), whose structures are maintained by EPCS resident proteins and the cytoskeleton [1-7] . Strong co-alignment between EPCS and the cytoskeleton is observed in plants $[1,8]$, but little is known of how the cytoskeleton is maintained and regulated at the EPCS. Here we have used a yeast-two-hybrid screen and subsequent in vivo interaction studies in plants by FRET-FLIM analysis, to identify two microtubule binding proteins, KLCR1 (Kinesin Light Chain Related protein 1) and IQD2 (IQ67-Domain 2) that interact with the actin binding protein NET3C and form a component of plant EPCS, that mediates the link between the actin and microtubule networks. The NET3C-KLCR1-IQD2 module, acting as an actin-microtubule bridging complex, has a direct influence on ER morphology and EPCS structure. Their loss of function mutants, net3a/NET3C RNAi, klcr1 or iqd2, exhibit defects in pavement cell morphology which we suggest is linked to the disorganization of both actin filaments and microtubules. In conclusion, our results reveal a novel cytoskeletal associated complex, which is essential for the maintenance and organization of cytoskeletal structure and ER morphology at the EPCS, and for normal plant cell morphogenesis. 


\section{Results and Discussion}

\section{Kinesin-light chain related protein (KLCR) interacts with NET3C}

NET3C belongs to a plant specific NETWORKED superfamily of actin binding proteins [9]; it localizes at the EPCS and interacts with the ER localized VAP27 proteins and the cytoskeleton $[1,10]$. In order to characterize the function of NET3C, a yeast-two-hybrid screen was performed using NET3C as bait, which identified an interaction with KLCR1 (Figure 1A). Previous studies have reported that KLCR1 (also referred to as Cellulose Microtubule Uncoupling 1), associates with microtubules and the plasma membrane and is predicted to stabilize microtubules at the cell cortex [11-13]. In Arabidopsis, KLCR1 has two close homologues (Figure 1B). One-on-one yeast-2-hybrid interaction tests were performed between NET3 and KLCR families. The results indicate that NET3C is able to interact with all three KLCR proteins, while NET3A exhibits weak interactions with both KLCR1 and 2 (Figure $1 C)$, suggesting some redundancy in their interactions.

In order to further investigate the interaction between NET3C and KLCR proteins, in vivo colocalization analyses were performed. In $N$. benthamiana leaf epidermal cells, RFP-NET3C is able to recruit KLCR1/2-GFP to the EPCS from the cytoplasm, but not KLCR3 (Figures 1D-E). The effectiveness of NET3C recruitment was measured using the signal intensity ratio; the strongest recruitment was seen with KLCR1 (Figure $1 \mathrm{H}$ ). In agreement with the co-localization results, the interaction between NET3C and KLCR1 was further confirmed in a FRET-FLIM assay. The average fluorescence lifetimes of GFP-NET3C was reduced significantly in the presence of KLCR1/2-RFP indicating strong physical interactions. Whereas, the negative control using free RFP is not able to reduce the lifetime of GFP-NET3C.

Taken together, we have identified an interaction between NET3C and KLCR1. Having such an interaction would enable KLCR1 to be recruited to VAP27/NET3C labelled EPCS.

KLCR1 forms stationary foci that associate with microtubules, ER and NET3C at endogenous conditions.

Analysis of subcellular KLCR1-GFP localization expressed in Arabidopsis at endogenous levels (driven by its native promoter in the klcr1 mutant background) revealed that KLCR1-GFP form stationary puncta that associate with cortical microtubules in a beads-on-a-string pattern (Figure 2A-2C). Most of these KLCR1 foci also associate with the ER; kymograph analyses indicate that these structures are relatively immobile and closely associate with stationary ER 
structures, a feature that is reminiscent of ER-PM contact sites (Figure 2D-2E).

To further confirm that KLCR1 foci are EPCS related, immunofluorescence studies were performed using the transgenic $p K L C R 1$ :KCLR1-GFP (Klcr1) line and a NET3C antibody that we have described previously [1]. As expected, endogenous NET3C localizes to EPCS that are associated with the ER network (Figure 2F). Strong co-alignment between endogenous NET3C and KLCR1-GFP labeled microtubules was identified; partial co-localization between NET3C and KLCR1 foci was also prominent (Figure 2G). Taken together, the co-localization results between anti-NET3C and ER; anti-NET3C and KLCR1-GFP, further support the conclusion that the KLCR1 labelled foci are EPCS associated. As control, the NET3C antibody was pre-incubated with a NET3C peptide before immuno-labeling and this resulted in the NET3C signal being almost completely abolished, indicating the effectiveness of the NET3C antibody (Figure $2 \mathrm{H}$ ). These results further confirm co-localization and interaction between KLCR1 and NET3C at endogenous expression levels.

\section{All three KLCRs associate with microtubules through IQ67 domain proteins (IQD)}

Previous studies reported that KLCR1 locates to microtubules through IQD proteins, which are plant-specific proteins that bind to calmodulin $[11,12]$. The IQD protein family has 33 members, most of them have been shown to localize to microtubules and regulate plant development [14-16]. IQD13is also able to associate with the plasma membrane and act as a lateral fence to spatially control the plasma membrane domains [17].

In this study, we further demonstrate that all three KLCR proteins can be recruited to microtubules through their interaction with IQD2 (Figure S1). When RFP tagged KLCR proteins are expressed alone in $N$. benthamiana, they show a cytosolic localization (Figures S1A-S1C). However, when they are co-expressed with GFP-IQD2, they can be clearly seen on the microtubules, indicating that the microtubule localization of KLCR proteins is dependent on IQD proteins (Figures S1D-S1G).

Upon strong expression, using the $35 \mathrm{~S}$ promoter to drive expression in $N$. benthamiana, KLCR proteins localize to the cytosol, indicating that the amount of endogenous IQD protein is too low to efficiently recruit large amounts of KLCR proteins to microtubules; the cytoplasmic background is also likely to mask any observable weak microtubule labeling. This phenomenon is similar to the localization patterns observed with TON1 which also localizes to the cytosol in tobacco cells but can be targeted to microtubules in the presence of TRM proteins $[18,19]$. However, when there is weak expression of KLCRs under the control of an ubiquitin promoter 
in $N$. benthamiana, co-alignment of KLCRs with microtubules is also observed (Figure S1H), similar to what is shown in stable transgenic lines.

\section{The NET3C-KLCR-IQD complex exists in plants}

As KLCR proteins are recruited to microtubules by proteins from the IQD family (Figure 2 and Figure S1), and KLCRs interact with NET3C at the ER-PM contact sites, we asked whether NET3C could also interact with IQDs to form a trimeric NET3C-KLCR-IQD complex, using the splitubiquitin system [20]. We observed no direct interaction between NET3C and IQD2 in this assay, but the interaction between KLCR1 and NET3C, and between KLCR1 and IQD2 was consistently reproducable (Figure 3A). Interestingly, when either KLCR1 or KLCR2 is included, interactions between NET3C and IQD2 are clearly identified (Figure 3B). Furthermore, almost complete co-localization was observed when these three proteins are co-expressed in vivo (Figure 3C).

In agreement with this result, we found that if the expression level of IQD2 is low (using low density Agrobacteria for infiltration), partial association between NET3C and IQD2 and microtubule is seen (Figure S2A), and the co-localization between NET3C and IQD2 can be enhanced in the presence of KLCR1 (Figure S2B). A strong association between NET3C and IQD2 is achieved when the expression levels of both NET3C and IQD2 are high (Figure S2C). It is likely that NET3C and IQD2 may exhibit an indirect or weak association, and KLCR acts as a 'linker/stabilizer' between NET3C and IQD2, facilitating the interaction. Similarly, partial association between GFP-IQD2 and endogenous NET3C is found in Arabidopsis cells that are immuno-labelled with the NET3C antibody (Figure S3A), whereas, complete association of IQD2 and NET3C can be achieved in Arabidopsis cells transiently over-expressing these proteins (Figure S3B). FRET-FLIM studies indicate that the fluorescence lifetime of GFP-NET3C is not affected dramatically when co-expressed with IQD2-RFP (despite strong co-localization), supporting the fact that these two proteins are port of a complex but do not directly interact (Figure 3D).

Moreover, when NET3C is co-expressed with other IQD proteins, clear co-localization can be observed with IQD1, IQD12 and IQD22, but not with IQD25 (Figure S4), suggesting that the complex of NET3C and IQDs exist across the different IQD subgroups. In summary, we have demonstrated the existence of a novel protein complex consisting of NET3C-KLCR1/2-IQD2 that crosslink actin and microtubules at the ER and PM interface $[1,11,14]$ (Figure 3Eand 3F). 


\section{at the EPCS}

It has been shown that alteration in EPCS structure or its protein composition has a direct effect on the morphology of the ER. For example, wider ER polygonal structures are observed in Arabidopsis T-DNA mutants where SYT1 is knocked out [2]. Moreover, ionic stress is able to redistribute SYT1 along cortical ER tubules and increases the number of EPCS tethers, thereby increasing ER-PM connectivity [3]. NET3C is a plant specific protein that mediates the link between the ER and the PM [1]. Therefore, altering the abundance and localization of NET3C may be predicted to have a direct impact on the morphology of the ER network. Indeed, the number of fully enclosed ER polygons is significantly increased in cells overexpressing NET3C $(p=9.1815 e-04)$ (Figure 3G-I). This is likely to be because NET3C overexpression results in the generation of additional ER-PM contact sites, thereby increasing the number of ER polygons (Figure 3J).

Endogenous NET3C localizes to discrete punctate structures which are closely associated with the ER network and KLCR1/IQD2 labelled microtubules (Figure 2F-G). Interestingly, when both RFP-NET3C and GFP-IQD2 are co-expressed in N. benthamiana, the overall ER network follows the same pattern as the microtubules (Figure 3K). Strong ER-microtubule co-alignment was found in cells co-expressing NET3C, IQD2 and VAP27-1; with NET3C/VAP27-1 labeled EPCS as puncta aligning along IQD2 labeled microtubules (Figure 3L). Therefore, the rearrangement of the ER network along microtubules is likely to be due to an increased ER-microtubule association, and the altered distribution of EPCS (Figure 3M). In addition, some triple transient expression studies have been performed as controls: NET3C, KLCR1 and VAP27-1 (Figure S5A); NET3C, KLCR1 and HDEL (Figure S5B); IQD2, KLCR1 and VAP27-1 (Figure S5C); IQD2, KLCR1 and HDEL (Figure S5D). None of these combinations exhibit ER-microtubule co-alignment as observed in Figure 3K. We therefore hypothesize that the co-existence of NET3C, VAP27, KLCR1 and IQD is important for mediating the interaction between ER-PM and the cytoskeleton.

As KLCR1 and IQD2 have been shown to localize to microtubules, and EPCS localized NET3C is known to bind the actin, then the NET3C-KLCR1-IQD2 complex could mediate the link between the two filament systems at the EPCS. To test this hypothesis, YFP-Actin-Cb or KMD-RFP were co-expressed with both IQD2 and NET3C in N. benthamiana. CFP-IQD2 co-localized with RFPNET3C on KMD-GFP labeled microtubules (Figure S5E) or YFP-Actin-Cb labeled actin filaments (Figure S5F). Cells expressing both YFP-Actin-Cb, KMD-RFP and HDEL were used as a control and no co-alignment between actin filaments, microtubules and ER network was observed (Figure S5G). 
It is known that the ER dynamics in plants is also regulated by microtubules but the molecular regulators have not been identified to date). As we have demonstrated that a NET3C-KLCR1IQD2 complex is able to mediate the connection between actin filaments and microtubules, this suggests that plants may have a specific mechanism for enabling actin-microtubule and ER interactions. Furthermore, enhanced association of ER and the cytoskeleton is found when NET3C-KLCR1-IQD2 is over-expressed (Figure 3K) which is in agreement with our conclusion that these proteins have the capability to mediate the link between ER and the cytoskeletal network (Figure 3E-F). However, when these proteins are kept at endogenous levels, the complex is localized to the place where ER, PM, Actin and Microtubules normally converge, that is at the EPCS (Figure 3M) [10].

The structures of ER/EPCS and the cytoskeleton are affected when the NET3C-KLCR1-IQD2 complex is dysfunctional.

In order to study the function of the NET3C-KLCR1-IQD complex in plants, we used T-DNA insertion mutants of $k / c r 1$ (the key component of the complex) (Figure S6), and crossed them with MAP4-GFP/Tubulin-mCherry (microtubules) and Lifeact-GFP (Actin) Arabidopsis reporter lines in order to study the phenotype from a subcellular perspective. Clear disorganization of the cytoskeletal network is observed in the klcr1 mutants (Figure 4A-B). The microtubule fibril array anisotropy score was used to assess microtubules organization, the score is from 0 (no order) to 1 (ordered) [21]. The average anisotropy score in wild type cells is observed to be $0.14 \pm 0.005$, which was significantly higher than that in $k l c r 1$ mutants which was $0.11 \pm 0.005$, indicating that the microtubule arrays in cotyledon pavement cells of klcr1 are less ordered (Figure 4C-D). Mutants carrying the actin marker appeared to have fewer weakly labeled thin filaments and more thick bundled filaments (Figure 4E). The skewness of fluorescence intensity distribution was used to investigate the level of actin filament bundling. The average skewness value of actin filaments in wild type plants is observed to be $2.03 \pm 0.03$, which was significantly smaller than the value in the same cells in klcr1 plants (2.36 \pm 0.04$)$ (Figure 4F).

Moreover, the EPCS marker VAP27-1-YFP was also crossed into klcr1 mutants. We found that the ER structure was affected in the klcr1 mutant; the area of ER cisternae increased significantly as quantified using "AnalyzER" (Figure 4I-J). In plants co-expressing VAP27-1-YFP and Lifeact-GFP, co-localization between thick ER streaming and actin bundles was prominent, and such an arrangement is rarely observed in wild type plants (Figure 4G-H). Interestingly, the size of VAP27 labelled EPCS appear much smaller (Figure 4I,K). Taken together, these data suggest that the NET3C-KLCR1-IQD complex is essential for EPCS structure. Interestingly, our 
previous study indicated that microtubules are essential for EPCS-VAP27 protein dynamics [1], which likely involves functions of microtubule-localized proteins at EPCS (e.g. KLCR1).

\section{Arabidopsis mutants of NET3C, KLCR1 and IQD2 are defective in cell expansion and pavement cell morphogenesis.}

When $k l c r 1$ and iqd 2 seedlings were grown on vertical plates, the overall development appears similar to wild type, however, their roots skewed to the left (Figure 4L-M). This phenotype is likely due to the left-handed helical arrangement of the root epidermal cells (Figure $4 \mathrm{~N}$ ), a phenomenon that has been reported in plants with aberrant microtubule organization [22-24]. Expression of proKLCR1:KLCR1-GFP or proIQD2:IQD2-GFP in their respective mutant plants was able to rescue the twisting root growth phenotype (Figure $4 \mathrm{~L}-\mathrm{N}$ ), suggesting that the phenotype of $k / c r 1$ and iqd2 is directly related to the loss-of-function of KLCR1 or IQD2.

Furthermore, quantitative analysis (using PaCeQuant [25]) suggests that the morphogenesis of leaf pavement cells is also significantly altered. In wild type plants, cotyledon pavement cells show an interlocking jigsaw puzzle appearance with lobes and indentations. However, in both the $k / c r 1$ and the iqd2 mutants, the cells became more circular, and other key parameters such as average lobe length and the max core width of the pavement cells are also significantly changed (Figure 4O-P). Moreover, pavement cell morphology in the net3a/NET3C RNAi mutant was also studied, as our result showed that both NET3A and NET3C are likely to interact with KLCR proteins (Figure 1C). The leaf pavement cells from these double mutants also exhibit a small but significant difference in cell area and circularity (Figure S7). Taken together, we suggest that disruption of the protein composition of the NET3C-KLCR1-IQD complex has an overall effect on cell morphology.

Some cytoskeletal associated proteins localize to the membrane-cytoskeleton interface [26, 27], where membrane remodeling could possibly interplay with cytoskeletal organization thereby regulating key subcellular activities. Members of the KLCR and IQD families were identified to be PM peripheral associated proteins from previous studies [12, 14]. Here we have demonstrated that these proteins can localize to the EPCS by interacting with NET3C, forming a NET3C-KLCR-IQD complex at the ER-PM interface where the actin cytoskeleton and microtubules converge. These findings support our hypothesis that EPCS in plants may act as a hub for cytoskeletal organization and signal sensing [28]. In the wild type, some actin cytoskeleton is cross-linked with microtubules at the EPCS $[1,10]$ through the NET3C-KLCR1IQD2 complex. The loss of function of KLCRs affects the association between NET3C and IQDs 
at the actin, microtubule and EPCS interface, resulting in changes in cytoskeletal and ER organization (Figure $4 Q$ ).

In plants with a dysfunctional NET3C-KLCR-IQD complex, the ER, EPCS structure and cytoskeletal organization are altered. As a consequence, a defective developmental and cell morphology phenotype was observed. However, with the tools we have to date, it is impossible to tell whether the mutant phenotype is a direct effect of cytoskeletal rearrangement, or ER/EPCS alteration. As the cytoskeleton-ER system are closely linked, affecting one component is likely to have a direct effect to the other. In previous work, IQD proteins have been shown to interact with calmodulin, which may suggest that the activity of the NET3C-KLCR1-IQD2 is mediated by a calcium signal. Therefore, it is possible that the EPCS associated NET3C-KLCR1-IQD2 complex is able to respond to certain stimuli that trigger intracellular calcium signals. Such calcium signals could be sensed by IQDs through their interaction with calmodulin, consequently changing cytoskeletal and ER organization in that region. This could be important as the degree of ER-PM connection has a direct effect on exocytosis, endocytosis, autophagy and lipid transport [29-33].

\section{Acknowledgements}

We thank Jinli Gong, Johan Kroon for their help in this work. The project was supported by NSFC grants (no. 91854102), Fundamental Research Funds for the Central Universities (2662018PY010), HZAU Scientific \& Technological Self-innovation Foundation (2017RC004) and Thousand Youth Talents Plan Project to P.W; and a BBSRC grant (BB/G006334/1) to P.J.H, and by GIF grant(no. G-1482-423.13/2018) and core funding of the Leibniz Association to K.B.

\section{Main Figures}




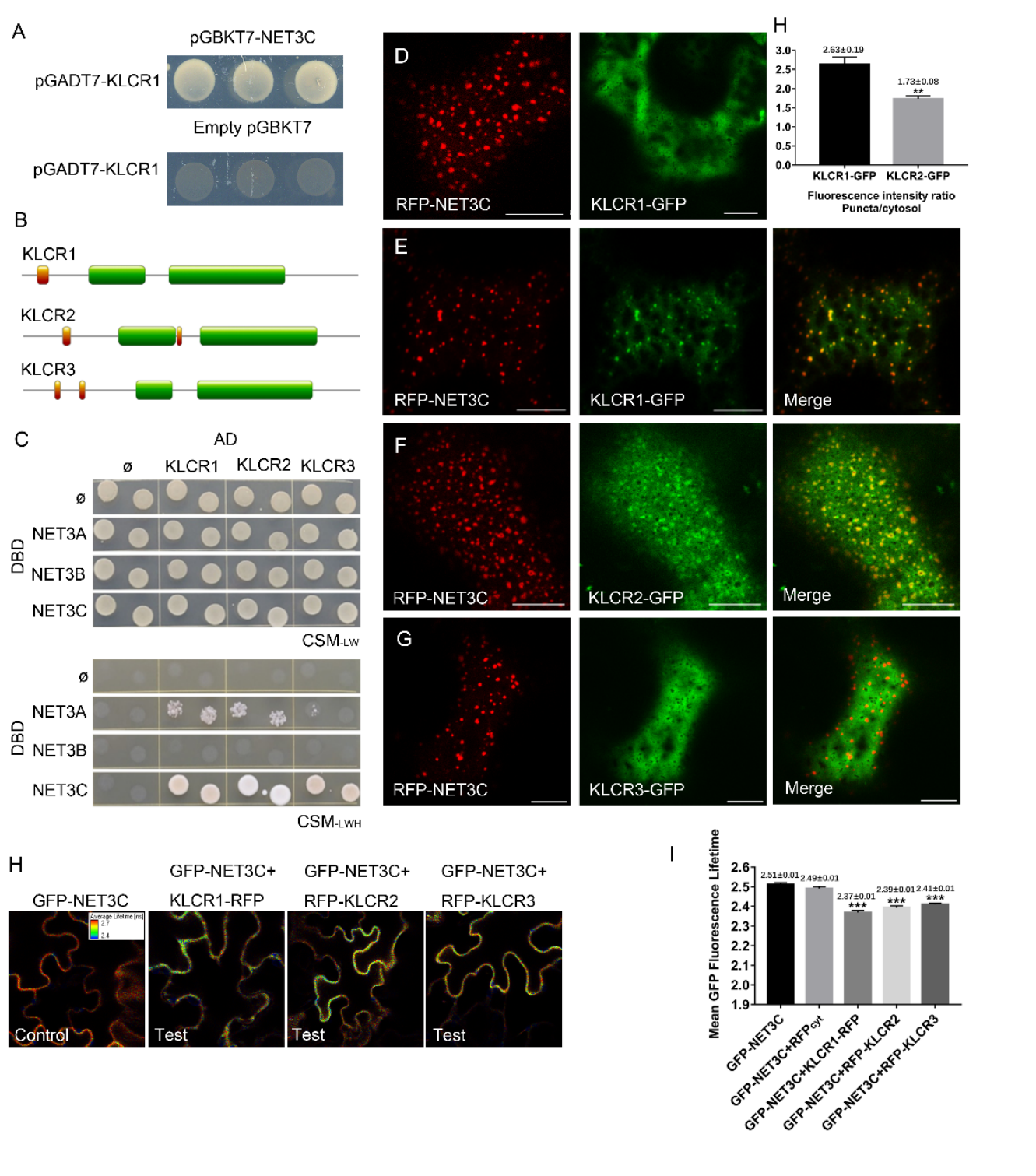

Figure 1. NET3C interacts with KLCR proteins. (A) The interaction between full length NET3C and KLCR (a.a.11-269) was identified in an Y2H screen. (B) Predicted protein structures of KLCR proteins in Arabidopsis using the SMART program (http://smart.embl-heidelberg.de/). Green boxes indicate tetratricopeptide regions (TPR), red boxes indicate regions of low complexity and grey lines represent non-domain containing protein regions. (C) $\mathrm{Y} 2 \mathrm{H}$ assay of interactions between NET3 proteins and full length KLCR proteins. Growth assay of haploid yeast cells coexpressing GAL4-DNA Binding Domain (DBD) fusions of NET3A, NET3B and NET3C with GAL4Activation Domain (AD) fusions of KLCR1, KLCR2 and KLCR3. DBD and AD alone $(\varnothing)$ were included as negative controls. Yeast cells were grown for 2 days on vector-selective media (CSM-LW) and on interaction-selective media (CSM-LWH). (D) The subcellular localization of NET3C and KLCR1 in N. benthamiana leaf tissues. NET3C and KLCR1 are localized at the punctate structures and in the cytosol, respectively, when they are expressed on their own. 
(E-G) The expression of RFP-NET3C is able to recruit KLCR1-GFP and KLCR2-GFP to punctate structures, but not KLCR3-GFP. (H-I) FRET-FLIM analysis of the interactions between NET3C, KLCR1, KLCR2 and KLCR3. The average fluorescence lifetime of GFP-NET3C when expressed on its own was found to be $2.51 \pm 0.01 \mathrm{~ns}$, whereas the average fluorescence lifetime of GFPNET3C was reduced by $0.14,0.12$ and 0.1 when co-expressed with KLCR1, KLCR2 and KLCR3 respectively. GFP-RFP was used as a positive control and the fluorescence lifetime was measured at $1.99 \pm 0.02 \mathrm{~ns}$. Scale bar: $10 \mu \mathrm{m}$.

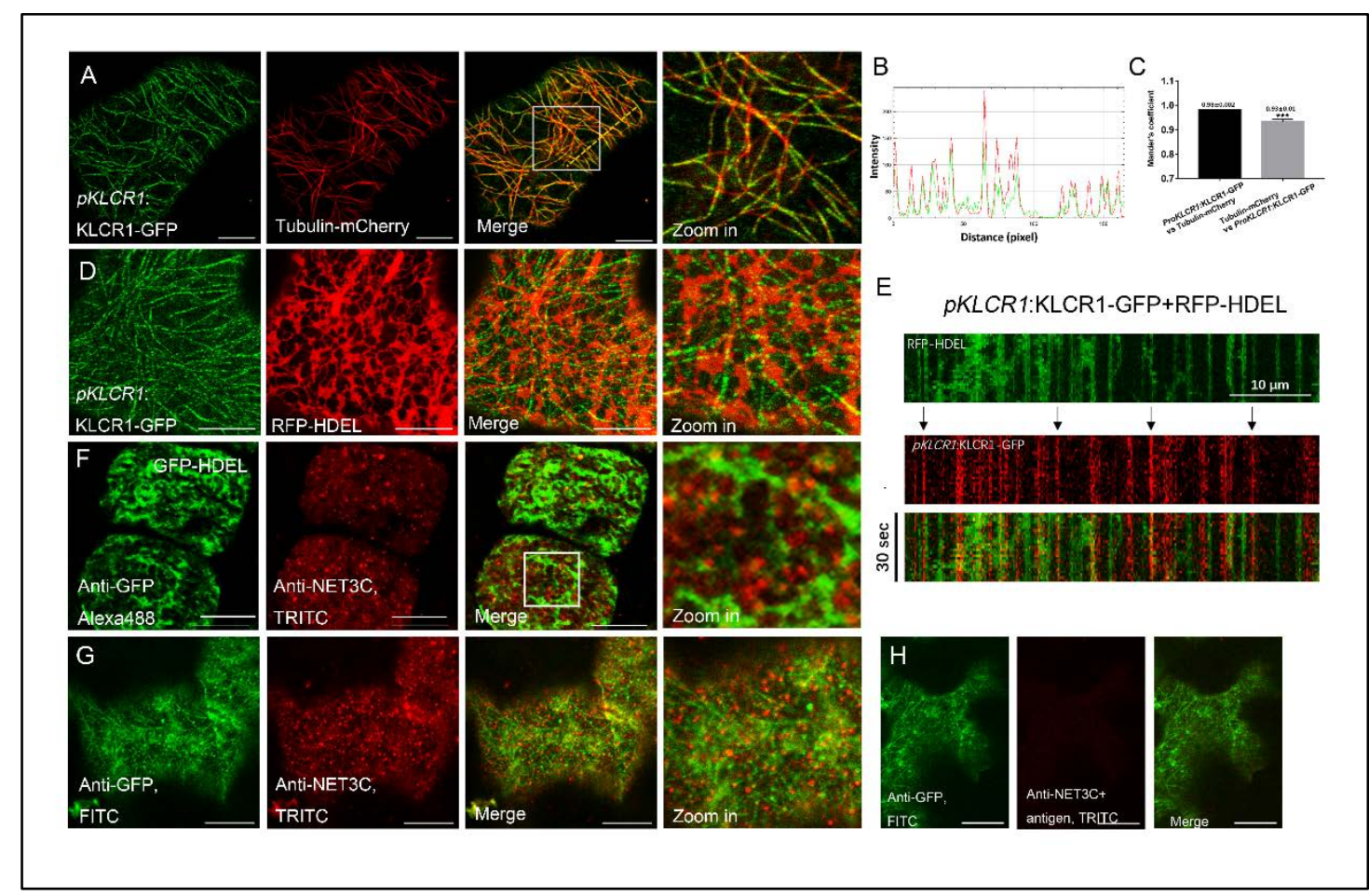

Figure 2. KLCR1 is associated with microtubules, ER and NET3C at the endogenous conditions.

(A) KLCR1 in Arabidopsis forms foci which co-localized with microtubules. (B) Fluorescence intensity plot along the dashed line in (A) (merge). The picks of KLCR1 and Tubulin signals were highly corelated. (C) Mander's coefficient test of KLCR1-GFP and Tubulin-mCherry colocalization in Arabidopsis cotyledon cells. The coefficient of the KLCR1-GFP against the Tubulin-mCherry is significantly higher than the reverse, indicating that not all of the microtubules were covered by KLCR1 signals. (D) Most of the KLCR1 foci are associated with the ER network. (E) Association of KLCR1 with the ER network further corroborated by a kymograph. The black arrows indicate the stable association sites. (F) Immunofluorescence of GFP-HDEL expressing Arabidopsis root tips with GFP and NET3C antibodies. Endogenous NET3C forms puncta which were associated with the ER network. (G) Immunofluorescence of pKLCR1:KLCR1-GFP expression Arabidopsis cotyledon epidermal cells with GFP and NET3C 
antibodies. Most of NET3C puncta co-aligned with KLCR1 labeled microtubules and colocalized with KLCR1 foci. (H) Co-incubation of the NET3C peptide immunogen with the NET3C antibody before immune-labeling. The NET3C signal seen previously was abolished with no effect on the KLCR1 labelling of the microtubules. Scale bar: $10 \mu \mathrm{m}$.

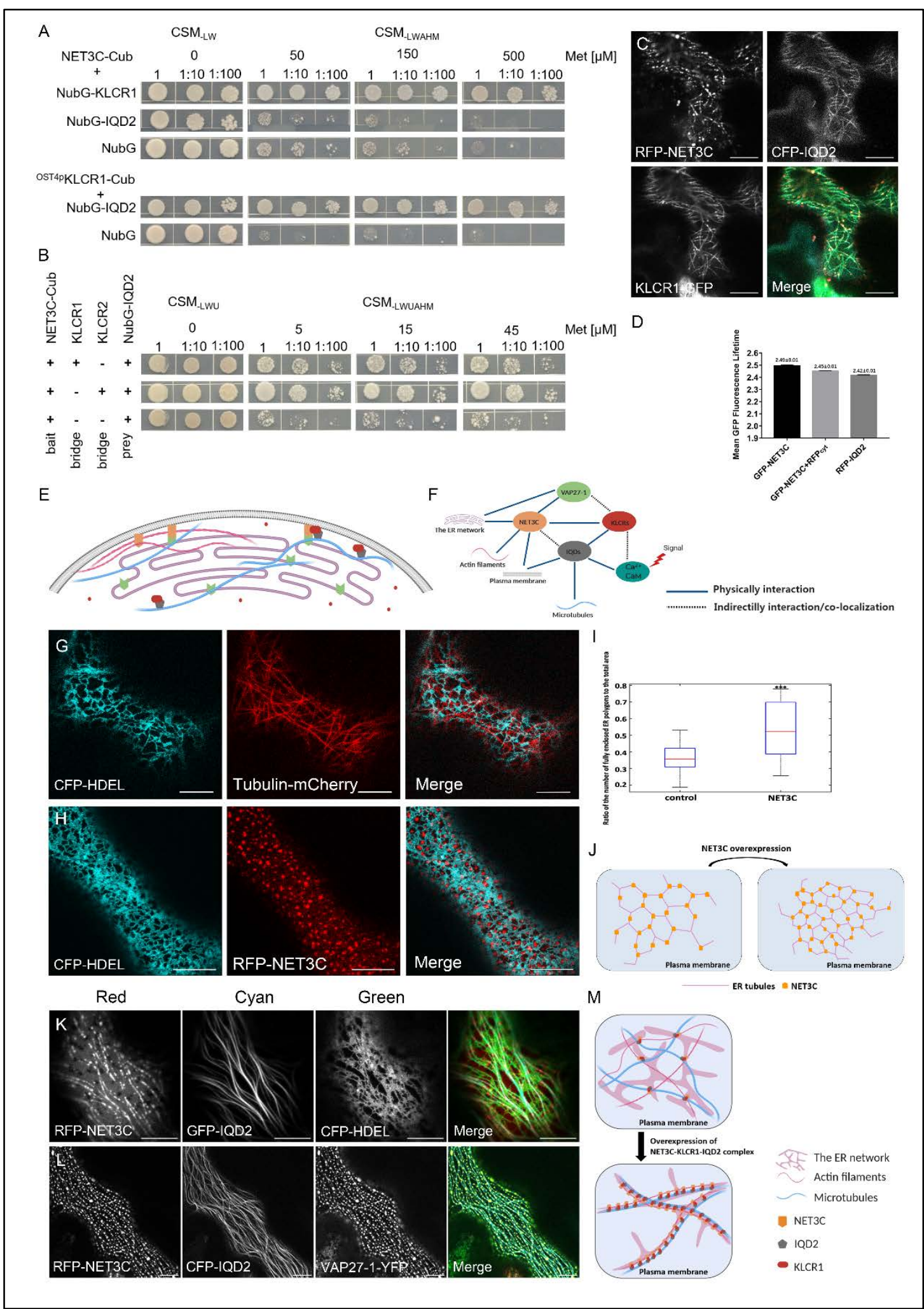


Figure 3. NET3C forms a complex with KLCR1 and IQD2, mediating the interaction between actin, microtubules and ER at the EPCS. (A) Growth assay of haploid yeast cells co-expressing NET3C-Cub with NubG-fusions of KLCR1 and IQD2 (top), and of KLCR1-Cub with NubG-IQD2 (bottom). NubG is included as a negative control; no direct interaction was observed after 3 days. (B) Growth assay of haploid yeast cells co-expressing the bait NET3C-Cub and the prey constructs Nub-IQD2. KLCR1 and KLCR2 were included as a linker in yeast cells in the top and middle rows, respectively. Yeast cells were used at OD600 value of $1(A)$ and $0.8(B)$, and in dilutions of 1:10 and 1:100 on vector selective media (A, CSM-LW, B, CSM-LWU) and on interaction media (A, CSM-LWAHM; B, CSM-LWUAHM) with increasing Met concentrations. Growth was monitored after 3 days (A) and 4 days (B). The results indicate that NET3C only interacts with IQD2 in the presence of KLCR1 and KLCR2, suggesting that KLCR proteins may function as a linker, facilitating the formation of a NET3C-KLCR-IQD complex. (C) The coexpression of RFP-NET3C, CFP-IQD2 and KLCR1-GFP in N. benthamiana suggest that the three proteins co-localized to microtubule like structures, and NET3C labeled EPCS as puncta aligning along microtubules. (D) (E) Putative model of NET3C-KLCRs-IQDs complex the EPCSs. KLCRs interact with IQDs at the microtubules and may transiently associated with EPCS and actin cytoskeleton through interacting with NET3C. (F) Diagram of interaction between IQDs, Net3C, KLCRs and VAP27-1. Blue solid lines indicate physical interactions which were confirmed using FRET-FLIM or yeast-two-hybrid. Dotted line indicates co-localization or indirect interactions. (G) N. benthamiana cells co-expressed with CFP-HDEL and TubulinmCherry were used as a control in the analysis of ER morphology. (H) The morphology of the ER (as labelled by GFP-HDEL) is altered when RFP-NET3C is over-expressed. The average number of polygonal ER structures were increased. (G-H, use black-white images as K-L). (I) The quantification of polygon numbers per micron. (J) Schematic of NET3C in relation to the cortical ER network. Under control conditions, the expression of NET3C is low and the ER meshwork appears to be normal. However, over-expression of NET3C may cause more ER-PM contact sites resulting in more ER polygonal rings being produced. (K-M) KLCR1, NET3C and IQD2 form a protein complex and facilitates the association between the ER network and microtubules. Over-expression of both IQD2 and NET3C induces a rearrangement of ER network along the microtubules. Scale bar: $10 \mu \mathrm{m}$. 


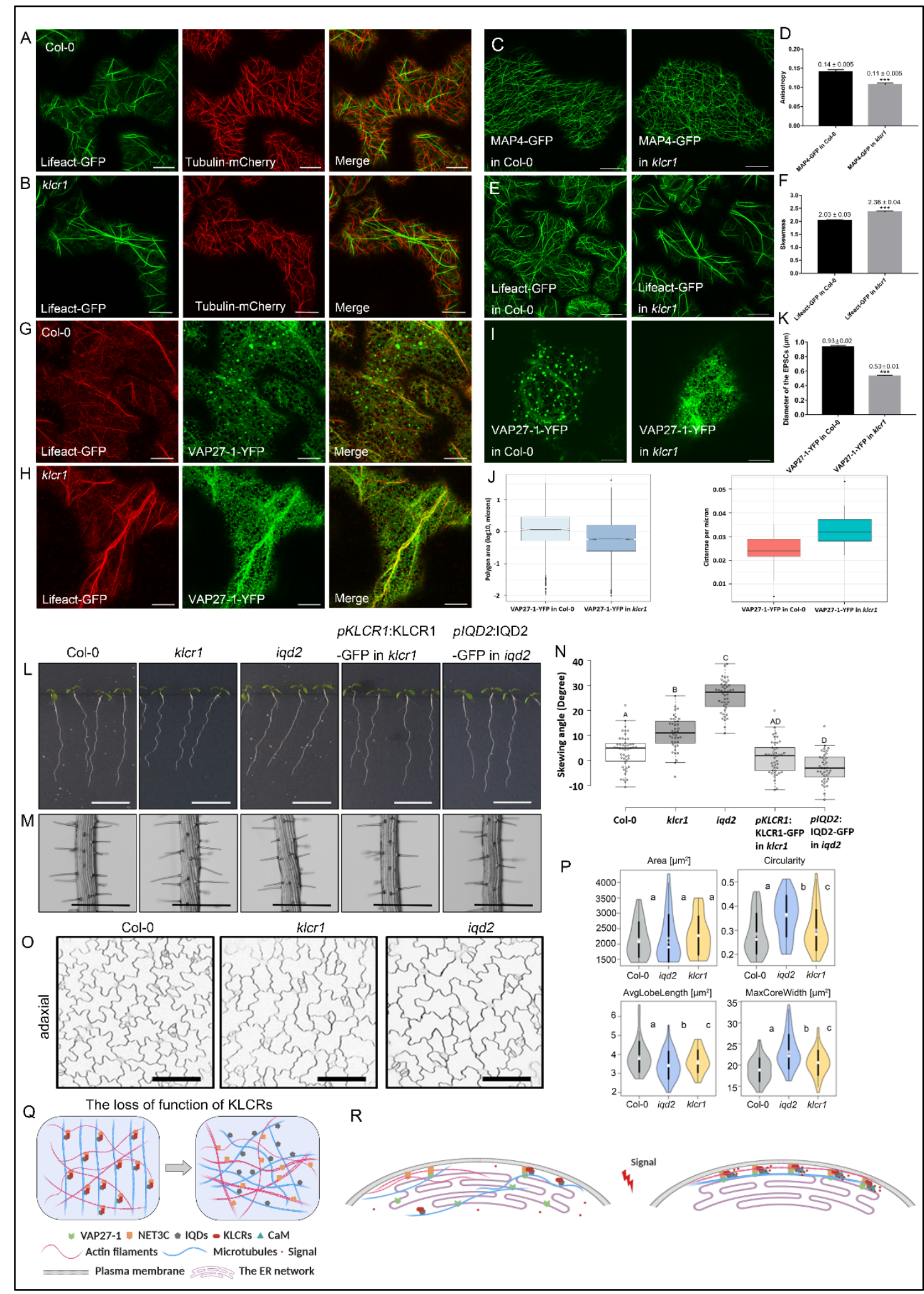

Figure 4. The loss of function mutants of KLCR1, NET3C and IQD2 are defective in pavement

cell morphogenesis. (A-B) Representative images of cotyledon pavement cells co-expressing Lifeact-GFP and Tubulin-mCherry. The cytoskeleton network is disorganized in the klcr1 mutants. (C-D) Representative images of cotyledon pavement cells expressing MAP4-GFP, quantitative analysis of cortical microtubule arrays suggested that the anisotropy of klcr1 
mutant was significantly reduced, suggesting the structure of microtubules are more disordered without KLCR1. (E-F) Representative images of cotyledon pavement cells expressing Lifeact-GFP, quantitative analysis suggesting that the actin filaments are more bunded (as measured by the skewness) in klcr1 mutant. Asterisks indicate statistically significant differences from wild type (Student's t-test, $\left.{ }^{* * *} p<0.001\right)$. (G)-(H) Representative images of cotyledon pavement cells co-expressing VAP27-1-YFP and Lifeact-GFP. Colocalization between thick ER stream and actin bundle can be clearly observed in klcr1 mutant. (I)-(J) Representative images of cotyledon pavement cells expressing VAP27-1-YFP. The structure of ER network and EPCSs were affected in klcr1 mutants. (L)-(M) Phenotypes of 7day-old Arabidopsis seedlings. Primary roots of klcr1 and iqd 2 mutants slanted towards the left side as viewed from above the plates. The root epidermal cells of klcrl and iqd2 showed left-handed helical arrangement when compared to wild type, whose root epidermal cells exhibited parallel arrangement. Expression of proKLCR1: KLCR1-GFP and proIQD2:IQD2-GFP in the $k l c r 1$ and iqd 2 mutants was able to rescue the twisting root growth phenotype. Root skewing scale bars: $1 \mathrm{~cm}$. Epidermal cell file rotation scale bars: $1 \mathrm{~mm}$. (N) Root slanting angles of 7-day-old wild type and mutant seedlings grown vertically on agar plates. (0) Pavement cell shape in cotyledon of 5-day-old seedlings. Inverted confocal images of the adaxial (top) side of cotyledons from wild type (Col-0) and klcr1 and iqd2 mutants stained with FM4-64. Scale bar: $100 \mu \mathrm{m}$. (P) Violin plots of value distributions of cell area, circularity, lobe length and maximum core width. Add info on $n=$ ? of analyzed cells. Circles and crosses refer to medians and means; vertical black lines represent SD (thick line) and the $95 \%$ confidence interval (thin lines). The width of each violin represents the local distribution of feature values along the $y$ axes. Different letters denote significant statistical differences. (Q) The loss of function of KLCR1 affects the structure of the cytoskeleton. 


\section{Supplementary Figure}

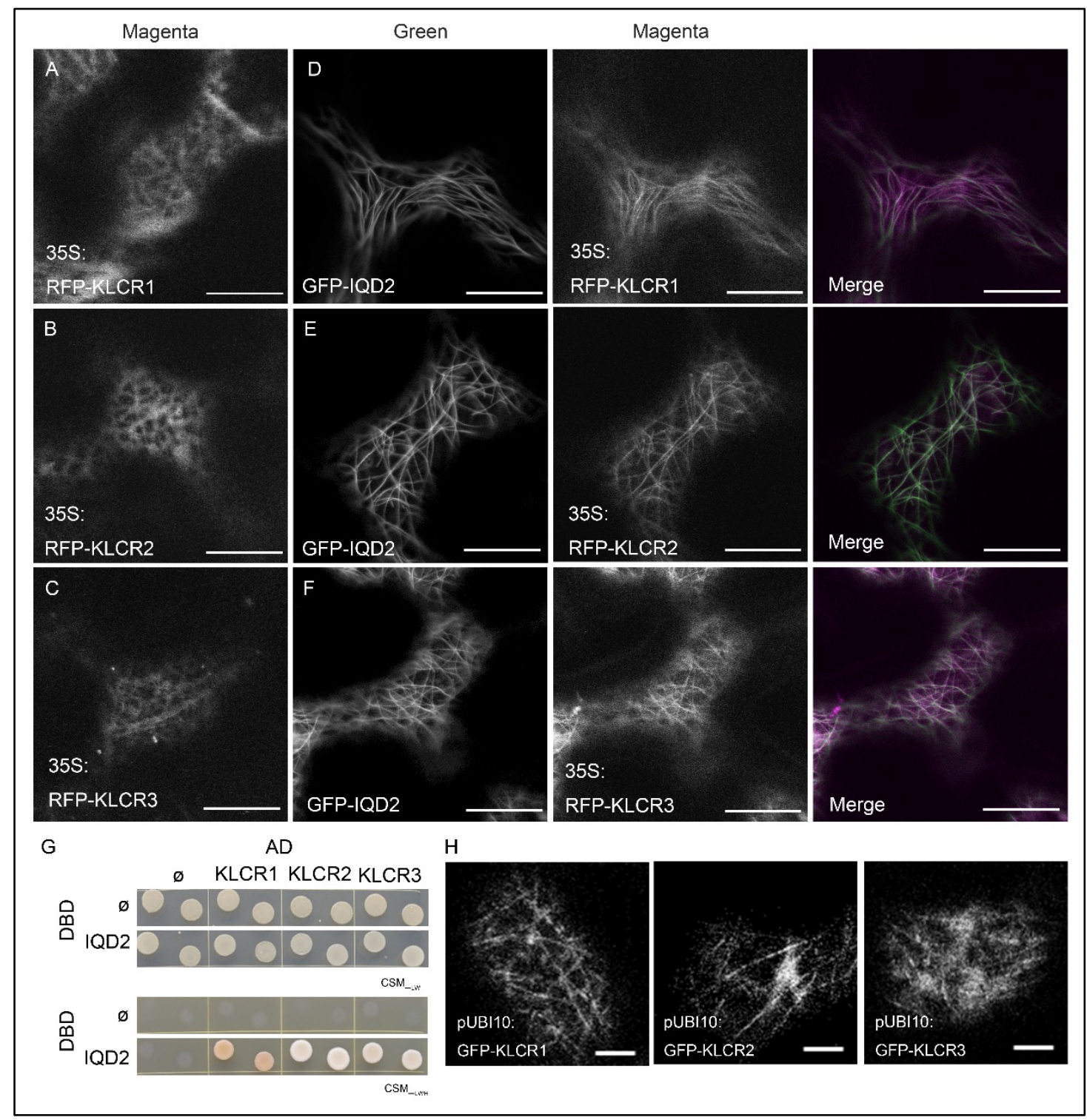

Figure S1. IQD2 interacts with KLCRs and recruits KLCRs to microtubules. (A)-(C) The localizations of KLCR1, KLCR2 and KLCR3 expressed under the control of $35 \mathrm{~S}$ promoter in N. benthamiana leaf epidermal cells. KLCRs localized to the cytosol. (D)-(F) Co-expression of GFPIQD2 with RFP-KLCR1, RFP-KLCR2 and RFP-KLCR3. RFP-KLCR1, RFP-KLCR2 and RFP-KLCR3 are recruited to microtubules by GFP-IQD2. Scale bar: $10 \mu \mathrm{m}$. (G) Growth assay of haploid yeast cells co-expressing DBD-IQD2 with AD-fusions KLCR1, KLCR2 and KLCR3. The results suggest interactions between these proteins. $(\mathrm{H})$ The localization of KLCR proteins expressed under the control of a weak promoter (Ubiquitin 10), the labelling of microtubule like structures is seen at the cell cortex. Scale bar: $5 \mu \mathrm{m}$. 


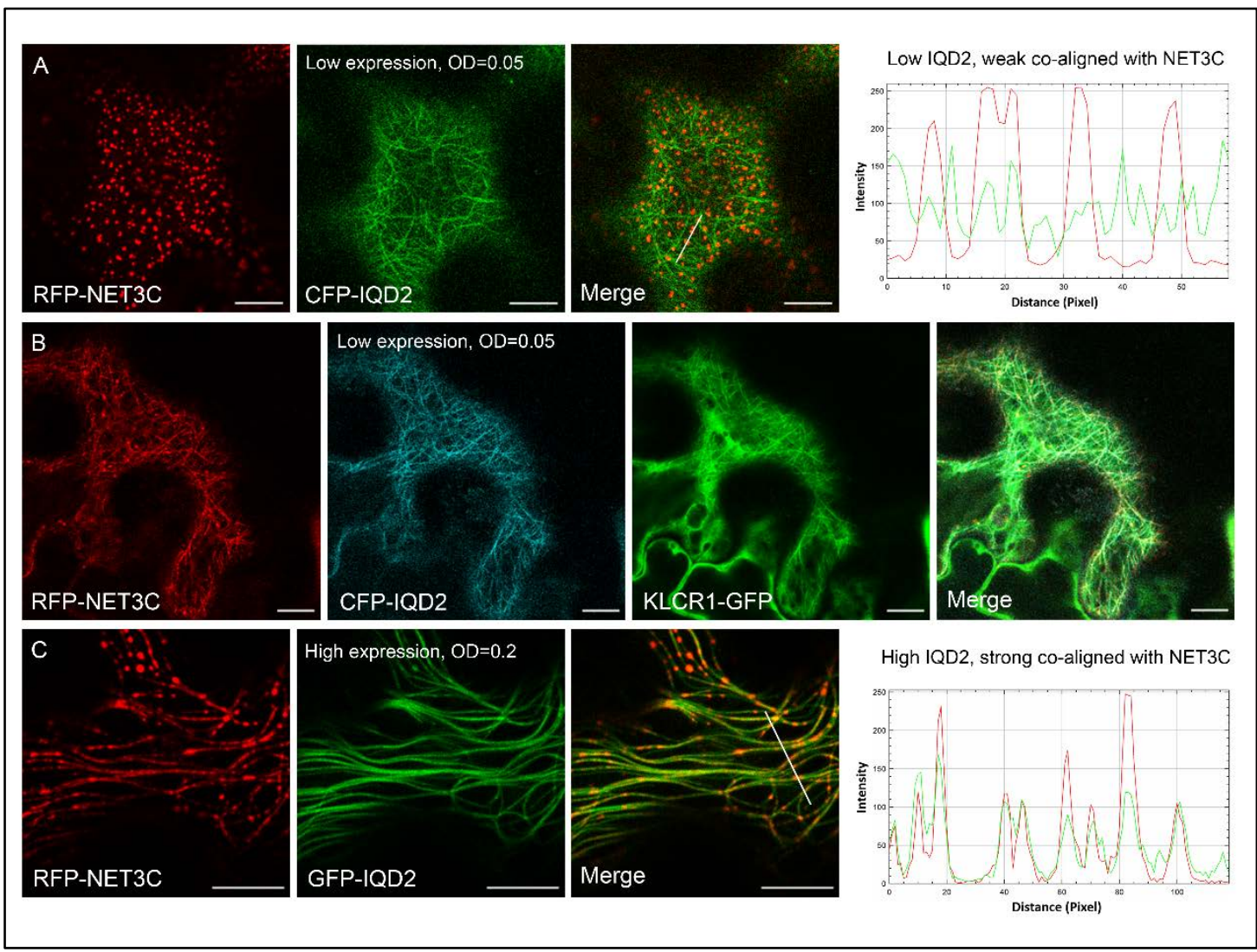

Figure S2. The interaction between NET3C and IQD2 depends on the presence of KLCR1. (A) Co-expression of RFP-NET3C with CFP-IQD2. CFP-IQD2 is not able to recruit RFP-NET3C to microtubules when its expression level is low. The fluorescence intensity plot along the dashed line in (A) (merge) indicates that the picks of NET3C and IQD2 signals were not corelated. (B) Co-expression of RFP-NET3C, CFP-IQD2 and KLCR1-GFP. RFP-NET3C can be recruited to microtubules in the presence of KLCR1. (C) Co-expression of RFP-NET3C with CFPIQD2 in high expression level. When the expression level of IQD2 is high, strong co-localization between RFP-NET3C and CFP-IQD2 can be observed. Scale bar: $10 \mu \mathrm{m}$. 


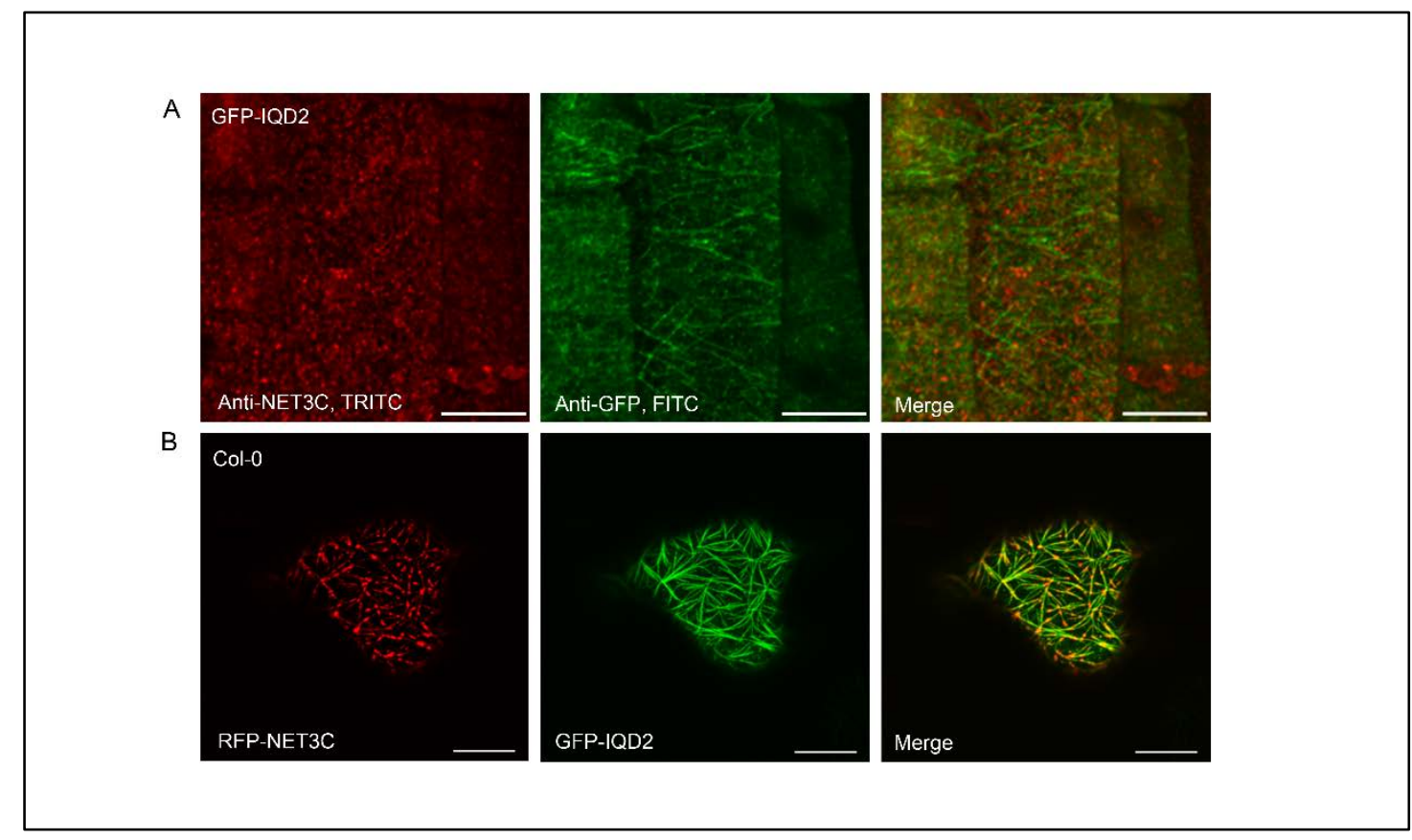

Figure S3. Endogenous NET3C is associated with IQD2. (A) Immunofluorescence of GFP-IQD2 expressing Arabidopsis root with GFP and NET3C antibody. Endogenous NET3C puncta coaligned with IQD2 labeled microtubules. (B) Transient co-expression of GFP-IQD2 with RFPNET3C in Arabidopsis cotyledon pavement cells. RFP-NET3C can be recruited to microtubules by GFP-IQD2. Scale bar: $10 \mu \mathrm{m}$. 

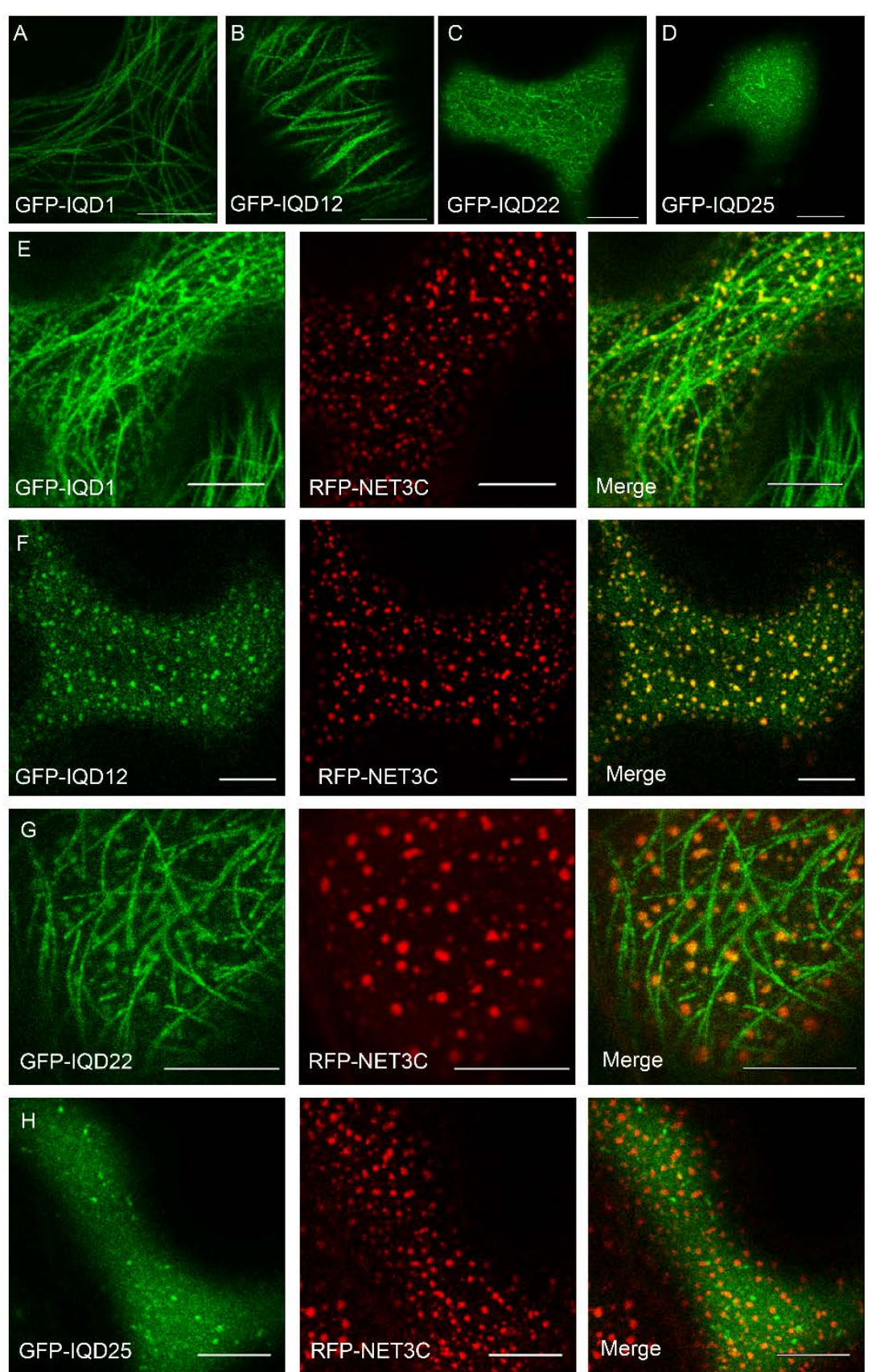

Figure S4. Co-localization study of NET3C with multiple IQD proteins in N. benthamiana leaf epidermal cells. (A)-(D) Subcellular localization of GFP-IQD1, GFP-IQD12, GFP-IQD22 and GFPIQD25 expressed on their own. (E) Co-expression of GFP-IQD1 with RFP-NET3C. GFP-IQD1 colocalizes with RFP-NET3C at punctate structures which associated with microtubules. (F) Coexpression of GFP-IQD12 with RFP-NET3C. GFP-IQD12 co-localizes with RFP-NET3C at punctate structures. (G) Co-expression of GFP-IQD22 with RFP-NET3C. GFP-IQD22 co-localizes with RFP-NET3C at punctate structures. (H) Co-expression of GFP-IQD25 with RFP-NET3C. GFPIQD25 does not co-localize with RFP-NET3C. (I) FRET-FLIM analysis of the interactions between NET3C and IQD1. Scale bar: $10 \mu \mathrm{m}$. 


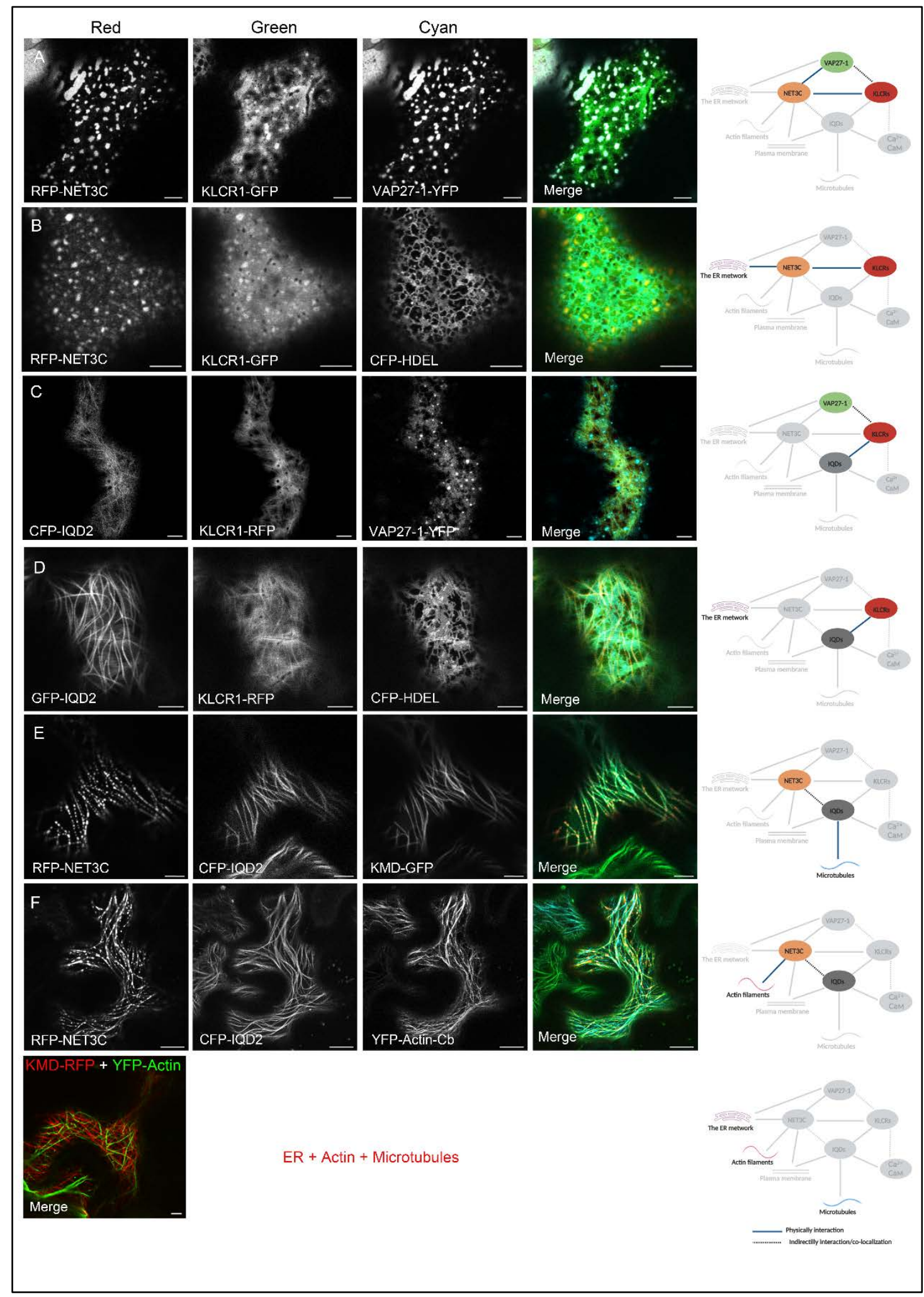

Figure S5. Triple expression analysis in $\mathbf{N}$. benthamiana leaf tissues. (A) Co-expression of RFPNET3C, KLCR1-GFP and VAP27-1-YFP. KLCR1-GFP can be recruited to NET3C and VAP27-1 labeled EPCSs. (B) Co-expression of RFP-NET3C, KLCR1-GFP and CFP-HDEL. (C) Co-expression of CFP-IQD2, KLCR1-RFP and VAP27-1-YFP. GFP-IQD2 and KLCR1-RFP do not co-localized woth VAP27-1 in the absence of NET3C. (D) Co-expression of GFP-IQD2, KLCR1-RFP and CFP-HDEL. 
(E) Co-expression of RFP-Net3C, CFP-IQD2 and KMD-GFP. RFP-NET3C is co-localized with CFPIQD2 at the microtubules. (F) Co-expression of RFP-NET3C, CFP-IQD2 and YFP-Actin-Cb. NET3C-KLCR1-IQD2 complex mediates the interaction between actin filaments and microtubules. (G) Co-expression of CFP-HDEL. Lifeact-GFP and KMD-RFP. None of the association between ER, actin filaments and microtubules can be observed. Scale bar: $10 \mu$ $\mathrm{m}$.

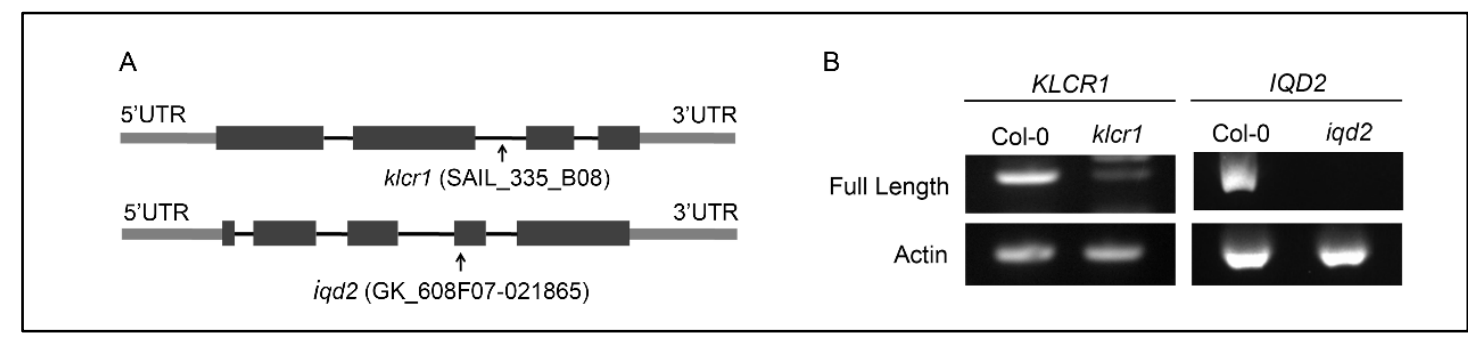

Figure S6. T-DNA insertion sites of klcr1 and iqd2. (A) Diagrammatic illustration of T-DNA insertion sites of klcr1 and iqd2. The genomic structure of KLCR1 and IQD2 was drawn to scale, the filled bars indicate Exons and introns are indicated by lines. The left borders of T-DNA insertion sites are indicated by arrows. (B) The expression levels of KLCR1 and IQD2 in their mutants were determined by RT-PCR.

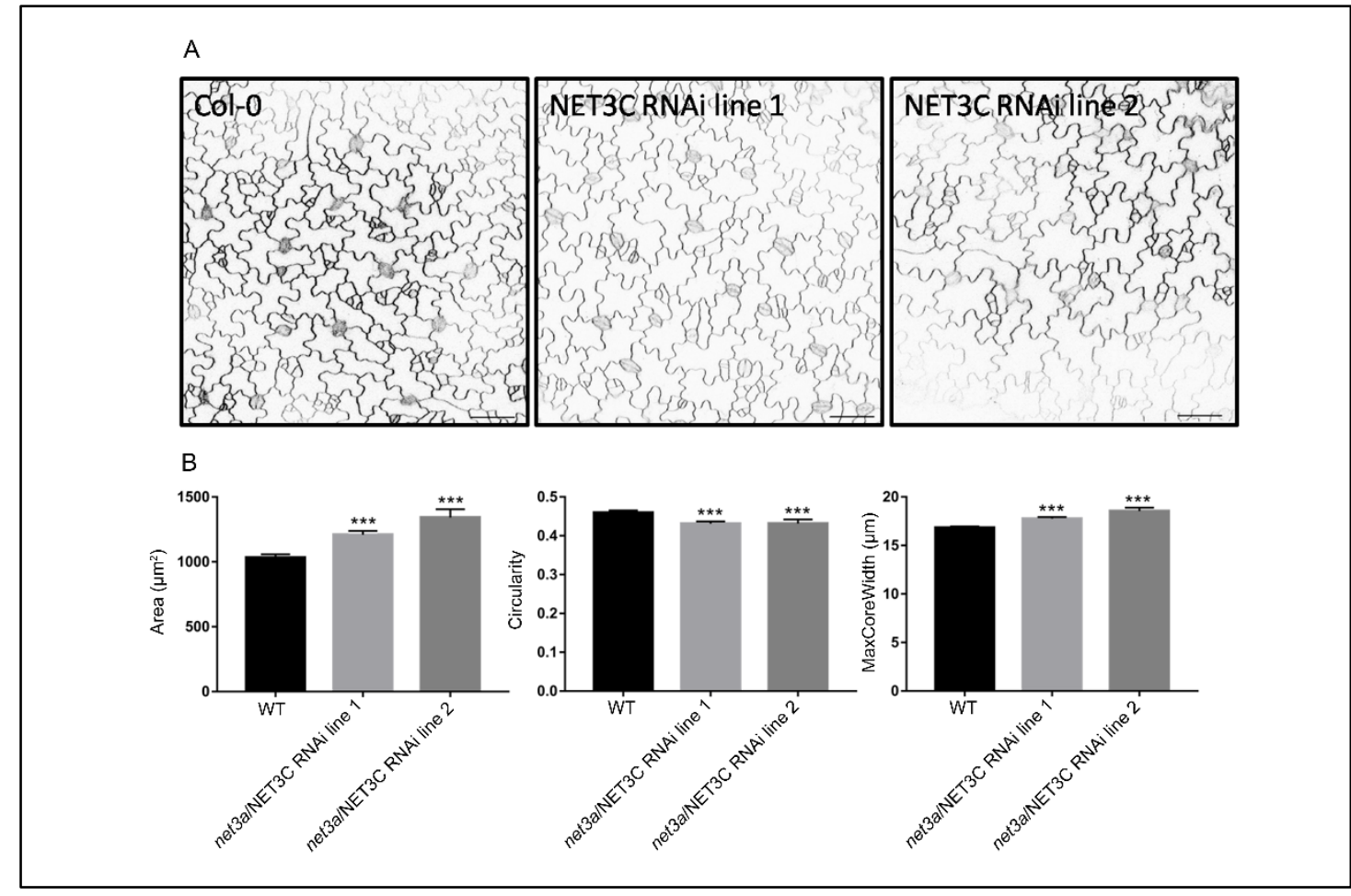

Figure S7. Pavement cell shape in cotyledon of 4-day-old seedlings. (A) Inverted confocal images of the adaxial (top) side of cotyledons from wild type (Col-0) and nat3a/NET3C RNAi mutants stained with FM4-64. (B) Quantitative analysis of net3a/NET3C RNAi pavement cell shape changes. 


\section{Reference}

1. Wang, P., Hawkins, T.J., Richardson, C., Cummins, I., Deeks, M.J., Sparkes, I., Hawes, C., and Hussey, P.J.J.C.B. (2014). The Plant Cytoskeleton, NET3C, and VAP27 Mediate the Link between the Plasma Membrane and Endoplasmic Reticulum. 24, 1397-1405.

2. Siao, W., Wang, P., Voigt, B., Hussey, P.J., and Baluska, F.J.J.o.E.B. (2016). Arabidopsis SYT1 maintains stability of cortical endoplasmic reticulum networks and VAP27-1-enriched endoplasmic reticulum-plasma membrane contact sites. 67, 6161-6171.

3. Lee, E., Vanneste, S., Pérez-Sancho, J., Benitez-Fuente, F., Strelau, M., Macho, A.P., Botella, M.A., Friml, J., and Rosado, A. (2019). Ionic stress enhances ERPM connectivity via phosphoinositide-associated SYT1 contact site expansion in Arabidopsis. Proc Natl Acad Sci U S A 116, 1420-1429.

4. Ishikawa, K., Tamura, K., Ueda, H., Ito, Y., Nakano, A., Hara-Nishimura, I., and Shimada, T. (2018). Synaptotagmin-Associated Endoplasmic ReticulumPlasma Membrane Contact Sites Are Localized to Immobile ER Tubules. Plant Physiol 178, 641-653.

5. Lee, E., Vila Nova Santana, B., Samuels, E., Benitez-Fuente, F., Corsi, E., Botella, M.A., Perez-Sancho, J., Vanneste, S., Friml, J., Macho, A., et al. (2020). Rare earth elements induce cytoskeleton-dependent and PI4P-associated rearrangement of SYT1/SYT5 ER-PM contact site complexes in Arabidopsis. J Exp Bot.

6. McFarlane, H.E., Lee, E.K., van Bezouwen, L.S., Ross, B., Rosado, A., and Samuels, A.L. (2017). Multiscale Structural Analysis of Plant ER-PM Contact Sites. Plant and Cell Physiology 58, 478-484.

7. Brault, M.L., Petit, J.D., Immel, F., Nicolas, W.J., Glavier, M., Brocard, L., Gaston, A., Fouché, M., Hawkins, T.J., Crowet, J.-M., et al. (2019). Multiple C2 domains and transmembrane region proteins (MCTPs) tether membranes at plasmodesmata. EMBO Rep 20, e47182.

8. Wang, P., Richardson, C., Hawkins, T.J., Sparkes, I., Hawes, C., and Hussey, P.J. (2016). Plant VAP27 proteins: domain characterization, intracellular localization and role in plant development. New Phytologist 210, 1311-1326.

9. Deeks, M.J., Calcutt, J.R., Ingle, E.K.S., Hawkins, T.J., Chapman, S., Richardson, A.C., Mentlak, D.A., Dixon, M.R., Cartwright, F., and Smertenko, A.J.C.B. (2012). A Superfamily of Actin-Binding Proteins at the ActinMembrane Nexus of Higher Plants. 22, 1595-1600.

10. Wang, P., Richardson, C., Hawkins, T.J., Sparkes, I., Hawes, C., and Hussey, P.J. (2016). Plant VAP27 proteins: domain characterization, intracellular localization and role in plant development. New Phytol 210, 1311-1326.

11. Burstenbinder, K., Savchenko, T., Muller, J., Adamson, A.W., Stamm, G., Kwong, R.W., Zipp, B.J., Dinesh, D.C., and Abel, S.J.J.o.B.C. (2013). Arabidopsis Calmodulin-binding Protein IQ67-Domain 1 Localizes to Microtubules and Interacts with Kinesin Light Chain-related Protein-1. 288, 1871-1882.

12. Liu, Z., Schneider, R., Kesten, C., Zhang, Y., Somssich, M., Zhang, Y., Fernie, A.R., and Persson, S.J.D.C. (2016). Cellulose-Microtubule Uncoupling Proteins Prevent Lateral Displacement of Microtubules during Cellulose Synthesis in Arabidopsis. 38, 305-315.

13. Ganguly, A., Zhu, C., Chen, W., and Dixit, R. (2020). FRA1 Kinesin Modulates the Lateral Stability of Cortical Microtubules through Cellulose Synthase- 
Microtubule Uncoupling Proteins. 32, 2508-2524.

14. Burstenbinder, K., Moller, B., Plotner, R., Stamm, G., Hause, G., Mitra, D., and Abel, S.J.P.P. (2017). The IQD Family of Calmodulin-Binding Proteins Links Calcium Signaling to Microtubules, Membrane Subdomains, and the Nucleus. 173, 1692-1708.

15. Liang, H., Zhang, Y., Martinez, P., Rasmussen, C.G., Xu, T., and Yang, Z.J.P.P. (2018). The Microtubule-Associated Protein IQ67 DOMAIN5 Modulates Microtubule Dynamics and Pavement Cell Shape. 177, 1555-1568.

16. Mitra, D., Klemm, S., Kumari, P., Quegwer, J., Moller, B., Poeschl, Y., Pflug, P., Stamm, G., Abel, S., and Burstenbinder, K.J.J.o.E.B. (2019). Microtubuleassociated protein IQ67 DOMAIN5 regulates morphogenesis of leaf pavement cells in Arabidopsis thaliana. 70, 529-543.

17. Sugiyama, Y., Wakazaki, M., Toyooka, K., Fukuda, H., and Oda, Y.J.C.B. (2017). A Novel Plasma Membrane-Anchored Protein Regulates Xylem CellWall Deposition through Microtubule-Dependent Lateral Inhibition of Rho GTPase Domains. 27, 2522.

18. Azimzadeh, J., Nacry, P., Christodoulidou, A., Drevensek, S., Camilleri, C., Amiour, N., Parcy, F., Pastuglia, M., and Bouchez, D.J.T.P.C. (2008). Arabidopsis TONNEAU1 Proteins Are Essential for Preprophase Band Formation and Interact with Centrin. 20, 2146-2159.

19. Drevensek, S., Goussot, M., Duroc, Y., Christodoulidou, A., Steyaert, S., Schaefer, E., Duvernois, E., Grandjean, O., Vantard, M., and Bouchez, D.J.T.P.C. (2012). The Arabidopsis TRM1-TON1 Interaction Reveals a Recruitment Network Common to Plant Cortical Microtubule Arrays and Eukaryotic Centrosomes. 24, 178-191.

20. Grefen, C., Obrdlik, P., and Harter, K.J.M.o.M.B. (2009). The determination of protein-protein interactions by the mating-based split-ubiquitin system (mbSUS). 479, 217-233.

21. Boudaoud, A., Burian, A., Borowskawykret, D., Uyttewaal, M., Wrzalik, R., Kwiatkowska, D., and Hamant, O.J.N.P. (2014). FibrilTool, an ImageJ plug-in to quantify fibrillar structures in raw microscopy images. 9, 457-463.

22. Furutani, I., Watanabe, Y., Prieto, R., Masukawa, M., Suzuki, K., Naoi, K., Thitamadee, S., Shikanai, T., and Hashimoto, T.J.D. (2000). The SPIRAL genes are required for directional control of cell elongation in Aarabidopsis thaliana. 127, 4443-4453.

23. Naoi, K., and Hashimoto, T.J.T.P.C. (2004). A Semidominant Mutation in an Arabidopsis Mitogen-Activated Protein Kinase Phosphatase-Like Gene Compromises Cortical Microtubule Organization. 16, 1841-1853.

24. Yuen, C.Y.L., Pearlman, R., Silosuh, L., Hilson, P., Carroll, K.L., and Masson, P.H.J.P.P. (2003). WVD2 and WDL1 Modulate Helical Organ Growth and Anisotropic Cell Expansion in Arabidopsis. 131, 493-506.

25. Moller, B., Poeschl, Y., Plotner, R., and Burstenbinder, K.J.P.P. (2017). PaCeQuant: A Tool for High-Throughput Quantification of Pavement Cell Shape Characteristics. 175, 998-1017.

26. Facette, M.R., Park, Y., Sutimantanapi, D., Luo, A., Cartwright, H.N., Yang, B., Bennett, E.J., Sylvester, A.W., and Smith, L.G.J.N.p. (2015). The SCAR/WAVE complex polarizes PAN receptors and promotes division asymmetry in maize. 1, 14024-14024.

27. Zhang, C., Mallery, E.L., and Szymanski, D.B.J.F.i.P.S. (2013). ARP2/3 localization in Arabidopsis leaf pavement cells: a diversity of intracellular pools 
and cytoskeletal interactions. 4, 238-238.

28. Wang, P., Hawes, C., and Hussey, P.J.J.T.i.P.S. (2017). Plant endoplasmic reticulum-plasma membrane contact sites. 22, 289-297.

29. Giordano, F., Saheki, Y., Idevallhagren, O., Colombo, S.F., Pirruccello, M., Milosevic, I., Gracheva, E.O., Bagriantsev, S.N., Borgese, N., and De Camilli, P.J.C. (2013). PI(4,5)P2-Dependent and Ca2+-Regulated ER-PM Interactions Mediated by the Extended Synaptotagmins. 153, 1494-1509.

30. Bayer, E.M., Sparkes, I., Vanneste, S., and Rosado, A.J.C.O.i.P.B. (2017). From shaping organelles to signalling platforms: the emerging functions of plant ERPM contact sites. 40, 89-96.

31. Basak, B., Krishnan, H., and Raghu, P.J.b. (2019). The lipid transfer function of RDGB at ER-PM contact sites is regulated by multiple interdomain interactions.

32. Ng, A.Y.E., Ng, A.Q.E., and Zhang, D.J.C.B. (2018). ER-PM Contacts Restrict Exocytic Sites for Polarized Morphogenesis. 28, 146.

33. Wang, P., Pleskot, R., Zang, J., Winkler, J., Wang, J., Yperman, K., Zhang, T., Wang, K., Gong, J., Guan, Y., et al. (2019). Plant AtEH/Pan1 proteins drive autophagosome formation at ER-PM contact sites with actin and endocytic machinery. In Nat Commun, Volume 10. p. 5132.

34. Wang, P., and Hussey, P.J.J.F.i.P.S. (2019). Plant ER-PM Contact Sites in Endocytosis and Autophagy: Does the Local Composition of Membrane Phospholipid Play a Role? 10, 23-23.

35. Stefano, G., Renna, L., Wormsbaecher, C., Gamble, J., Zienkiewicz, K., and Brandizzi, F. (2018). Plant Endocytosis Requires the ER Membrane-Anchored Proteins VAP27-1 and VAP27-3. Cell Rep 23, 2299-2307. 